\title{
Globalization of sponsored clinical trials
}

Johan P. E. Karlberg

We read with interest the recent article in Nature Reviews Drug Discovery on trends in the globalization of clinical trials ${ }^{1}$, as we have also analysed US clinical trial registry data (ClinicalTrials.gov) $^{2}$ to study the same topic using data downloaded on 4 April 2007.

We identified 188,428 study sites for 8,143 industry-sponsored Phase I-IV trials (recruiting, no longer recruiting/ongoing or completed), compared with 123,713 sites for 6,046 studies identified by Thiers and colleagues $^{1}$, and their paper was ultimately based on 74,499 study sites $(39.5 \%$ of all sites). Among different results obtained in our analysis of our larger data set, the United Kingdom ranked fifth with 4,931 sites, whereas REF. 1 ranked the country eighth with 1,753 sites.

The paper by Thiers et al. also ranked countries according to annual growth rate in the number of sites, based on studies registered between 2002 and 2006. However, the US trial register was not utilized on a large scale until the introduction of the International Committee of Medical Journal Editors (ICMJE) publication policy in July 2005 (REF. 3). Before mid-2005, only around 400 new industry-sponsored trials were on average registered annually, compared with 3,400 after mid-2005. Since mid-2005, 24 of the world's top 25 pharmaceutical companies have utilized the register, while several leading companies including Pfizer, AstraZeneca, Boehringer Ingelheim, Wyeth and Takeda rarely registered before this point in time. Furthermore, the majority of sites $(67.7 \% ; 40,654 / 60,037)$ registered before mid-2005 were US sites, compared with $46.9 \%(60,270 / 128,391)$ from mid-2005 onwards ${ }^{4}$. The number of study sites in the US thus increased by $143 \%$, while all other 49 top-ranked countries showed an increase of at least $200 \%$ - with 20 countries showing an increase of more than $400 \%$. Clearly, there was selective registration of trials with ClinicalTrials.gov prior to the implementation of the ICMJE policy in mid-2005.

We also downloaded the US trial register data on 1 November 2007 and kept for analysis industry-sponsored Phase II-IV trials (recruiting, no longer recruiting/ongoing or completed) with at least

\section{Top 50 countries}

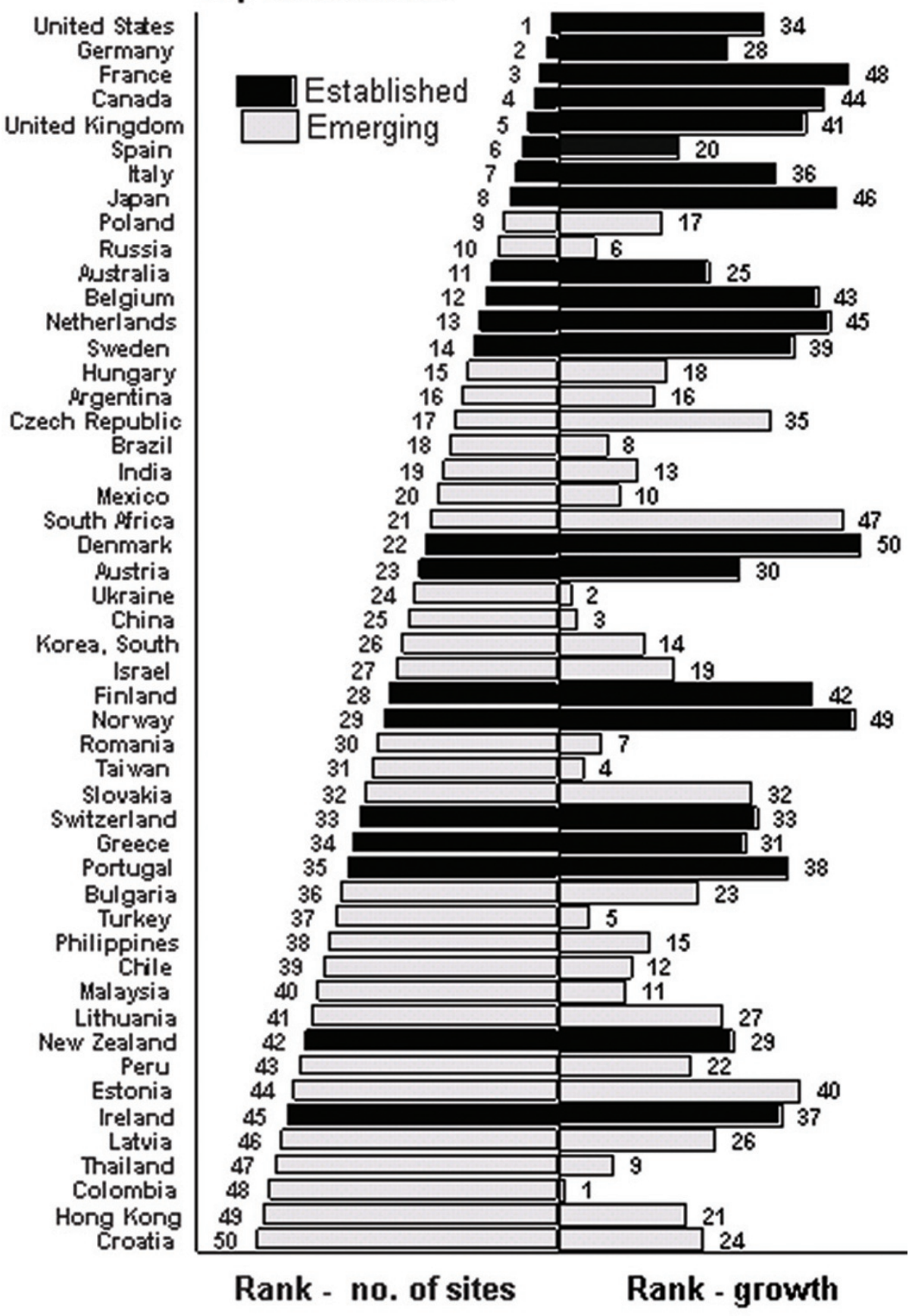

Figure 1 | The top 50 countries ranked by the total number of Phase II-IV study sites registered with ClinicalTrials.gov over a 24-month period from October 2005 to September 2007 (rank: number of sites). The annual growth in the number of study sites from the first year to the second year gives a second ranking (rank: growth). 


\begin{tabular}{|c|c|c|c|c|c|c|c|c|c|c|c|c|c|c|c|}
\hline Rank & Rank & Rank & & & & Yr 1 & $\operatorname{Yr} 1$ & Yr 2 & Yr 2 & Total & Total & Sites per & $\begin{array}{l}\text { GROWTH } \\
\text { Yr 1-Yr } 2^{\star}\end{array}$ & GROWTH & \\
\hline Total & Yr 1 & $\operatorname{Yr} 2$ & Change & & & Protocols & Sites & Protocols & Sites & Protocols & SITES & Protocol & Protocols & Sites & $\begin{array}{l}\text { Rank by } \\
\text { Sites }\end{array}$ \\
\hline SITES & Sites & Sites & Rank & Region & Country & $\mathrm{N}$ & $\mathrm{N}$ & $\mathrm{N}$ & $\mathrm{N}$ & $\mathrm{N}$ & $\mathrm{N}$ & $\mathrm{N}$ & $\%$ & $\%$ & $\%$ \\
\hline 1 & 1 & 1 & 0 & North America & United States & 1,388 & 34,131 & 1,382 & 24,802 & 2,770 & 58,933 & 21.3 & 99.6 & 72.7 & 34 \\
\hline 2 & 2 & 2 & 0 & Europe & Germany & 547 & 4,436 & 491 & 3,389 & 1,038 & 7,825 & 7.5 & 89.8 & 76.4 & 28 \\
\hline 3 & 3 & 4 & 1 & Europe & France & 406 & 4.213 & 303 & 1.834 & 709 & 6.047 & 8.5 & 74.6 & 43.5 & 48 \\
\hline 4 & 4 & 3 & -1 & North America & Canada & 440 & 3,493 & 325 & 1,870 & 765 & 5,363 & 7.0 & 73.9 & 53.5 & 44 \\
\hline 5 & 5 & 6 & 1 & Europe & $\begin{array}{l}\text { United } \\
\text { Kingdom }\end{array}$ & 378 & 2.389 & 307 & 1.373 & 685 & 3.762 & 5.5 & 81.2 & 57.5 & 41 \\
\hline 6 & 7 & 5 & -2 & Europe & Spain & 322 & 1,922 & 301 & 1,635 & 623 & 3,557 & 5.7 & 93.5 & 85.1 & 20 \\
\hline 7 & 6 & 7 & 1 & Europe & Italy & 312 & 1.945 & 274 & 1.355 & 586 & 3.300 & 5.6 & 87.8 & 69.7 & 36 \\
\hline 8 & 8 & 10 & 2 & Asia & Japan & 135 & 1,785 & 122 & 922 & 257 & 2,707 & 10.5 & 90.4 & 51.7 & 46 \\
\hline 9 & 9 & 9 & 0 & E. Europe \& Russia & Poland & 245 & 1.235 & 229 & 1.093 & 474 & 2,328 & 4.9 & 93.5 & 88.5 & 17 \\
\hline 10 & 11 & 8 & -3 & E. Europe \& Russia & Russia & 181 & 1,093 & 178 & 1,184 & 359 & 2,277 & 6.3 & 98.3 & 108.3 & 6 \\
\hline 11 & 12 & 11 & -1 & Oceania & Australia & 235 & 1,082 & 197 & 870 & 432 & 1,952 & 4.5 & 83.8 & 80.4 & 25 \\
\hline 12 & 10 & 12 & 2 & Europe & Belgium & 233 & 1,218 & 221 & 683 & 454 & 1,901 & 4.2 & 94.8 & 56.1 & 43 \\
\hline 13 & 13 & 18 & 5 & Europe & Netherlands & 247 & 1,078 & 178 & 574 & 425 & 1,652 & 3.9 & 72.1 & 53.2 & 45 \\
\hline 14 & 14 & 20 & 6 & Europe & Sweden & 184 & 911 & 162 & 529 & 346 & 1,440 & 4.2 & 88.0 & 58.1 & 39 \\
\hline 15 & 18 & 14 & -4 & E. Europe \& Russia & Hungary & 176 & 706 & 150 & 620 & 326 & 1,326 & 4.1 & 85.2 & 87.8 & 18 \\
\hline 16 & 19 & 15 & -4 & Latin America & Argentina & 146 & 691 & 137 & 620 & 283 & 1,311 & 4.6 & 93.8 & 89.7 & 16 \\
\hline 17 & 15 & 19 & 4 & E. Europe \& Russia & $\begin{array}{l}\text { Czech } \\
\text { Republic }\end{array}$ & 193 & 766 & 145 & 545 & 338 & 1,311 & 3.9 & 75.1 & 71.1 & 35 \\
\hline 18 & 21 & 13 & -8 & Latin America & Brazil & 128 & 627 & 122 & 629 & 250 & 1,256 & 5.0 & 95.3 & 100.3 & 8 \\
\hline 19 & 20 & 16 & -4 & Asia & India & 112 & 650 & 115 & 606 & 227 & 1,256 & 5.5 & 102.7 & 93.2 & 13 \\
\hline 20 & 22 & 17 & -5 & Latin America & Mexico & 155 & 601 & 153 & 581 & 308 & 1,182 & 3.8 & 98.7 & 96.7 & 10 \\
\hline 21 & 17 & 28 & 11 & Africa & South Africa & 171 & 709 & 100 & 323 & 271 & 1,032 & 3.8 & 58.5 & 45.6 & 47 \\
\hline 22 & 16 & 29 & 13 & Europe & Denmark & 166 & 725 & 125 & 297 & 291 & 1,022 & 3.5 & 75.3 & 41.0 & 50 \\
\hline 23 & 23 & 23 & 0 & Europe & Austria & 156 & 578 & 162 & 428 & 318 & 1,006 & 3.2 & 103.8 & 74.0 & 30 \\
\hline 24 & 28 & 21 & -7 & E. Europe $\&$ Russia & Ukraine & 62 & 388 & 72 & 501 & 134 & 889 & 6.6 & 116.1 & 129.1 & 2 \\
\hline 25 & 29 & 22 & -7 & Asia & China & 83 & 377 & 93 & 477 & 176 & 854 & 4.9 & 112.0 & 126.5 & 3 \\
\hline 26 & 27 & 24 & -3 & Asia & Korea, South & 112 & 439 & 122 & 406 & 234 & 845 & 3.6 & 108.9 & 92.5 & 14 \\
\hline 27 & 26 & 25 & -1 & Middle East & Israel & 117 & 451 & 106 & 390 & 223 & 841 & 3.8 & 90.6 & 86.5 & 19 \\
\hline 28 & 25 & 30 & 5 & Europe & Finland & 139 & 484 & 114 & 277 & 253 & 761 & 3.0 & 82.0 & 57.2 & 42 \\
\hline 29 & 24 & 35 & 11 & Europe & Norway & 105 & 503 & 72 & 207 & 177 & 710 & 4.0 & 68.6 & 41.2 & 49 \\
\hline 30 & 30 & 26 & -4 & $\begin{array}{l}\text { E. Europe \& } \\
\text { Russia }\end{array}$ & Romania & 100 & 349 & 86 & 352 & 186 & 701 & 3.8 & 86.0 & 100.9 & 7 \\
\hline 31 & 35 & 27 & -8 & Asia & Taiwan & 95 & 284 & 115 & 350 & 210 & 634 & 3.0 & 121.1 & 123.2 & 4 \\
\hline 32 & 31 & 31 & 0 & E. Europe \& Russia & Slovakia & 81 & 343 & 69 & 253 & 150 & 596 & 4.0 & 85.2 & 73.8 & 32 \\
\hline 33 & 32 & 32 & 0 & Europe & Switzerland & 143 & 326 & 117 & 238 & 260 & 564 & 2.2 & 81.8 & 73.0 & 33 \\
\hline 34 & 34 & 33 & -1 & Europe & Greece & 86 & 303 & 73 & 224 & 159 & 527 & 3.3 & 84.9 & 73.9 & 31 \\
\hline 35 & 33 & 36 & 3 & Europe & Portugal & 89 & 315 & 65 & 207 & 154 & 522 & 3.4 & 73.0 & 65.7 & 38 \\
\hline 36 & 36 & 34 & -2 & E. Europe \& Russia & Bulgaria & 72 & 262 & 52 & 214 & 124 & 476 & 3.8 & 72.2 & 81.7 & 23 \\
\hline 37 & 38 & 37 & -1 & E. Europe \& Russia & Turkey & 62 & 153 & 67 & 185 & 129 & 338 & 2.6 & 108.1 & 120.9 & 5 \\
\hline 38 & 37 & 38 & 1 & Asia & Philippines & 56 & 158 & 46 & 143 & 102 & 301 & 3.0 & 82.1 & 90.5 & 15 \\
\hline 39 & 39 & 39 & 0 & Latin America & Chile & 71 & 151 & 63 & 142 & 134 & 293 & 2.2 & 88.7 & 94.0 & 12 \\
\hline 40 & 44 & 41 & -3 & Asia & Malaysia & 47 & 131 & 52 & 126 & 99 & 257 & 2.6 & 110.6 & 96.2 & 11 \\
\hline 41 & 41 & 42 & 1 & E. Europe \& Russia & Lithuania & 47 & 140 & 50 & 111 & 97 & 251 & 2.6 & 106.4 & 79.3 & 27 \\
\hline 42 & 42 & 44 & 2 & Oceania & New Zealand & 59 & 135 & 47 & 102 & 106 & 237 & 2.2 & 79.7 & 75.6 & 29 \\
\hline 43 & 45 & 43 & -2 & Latin America & Peru & 51 & 129 & 44 & 107 & 95 & 236 & 2.5 & 86.3 & 82.9 & 22 \\
\hline 44 & 40 & 49 & 9 & E. Europe \& Russia & Estonia & 55 & 143 & 39 & 83 & 94 & 226 & 2.4 & 70.9 & 58.0 & 40 \\
\hline 45 & 43 & 46 & 3 & Europe & Ireland & 60 & 133 & 43 & 91 & 103 & 224 & 2.2 & 71.7 & 68.4 & 37 \\
\hline 46 & 46 & 47 & 1 & E. Europe \& Russia & Latvia & 38 & 114 & 42 & 91 & 80 & 205 & 2.6 & 110.5 & 79.8 & 26 \\
\hline 47 & 48 & 45 & -3 & Asia & Thailand & 55 & 102 & 44 & 102 & 99 & 204 & 2.1 & 80.0 & 100.0 & 9 \\
\hline 48 & 51 & 40 & -11 & Latin America & Colombia & 30 & 67 & 48 & 134 & 78 & 201 & 2.6 & 160.0 & 200.0 & 1 \\
\hline 49 & 47 & 48 & 1 & Asia & Hong Kong & 65 & 104 & 56 & 87 & 121 & 191 & 1.6 & 86.2 & 83.7 & 21 \\
\hline 50 & 49 & 51 & 2 & E. Europe $\varnothing$ Russia & Croatia & 43 & 94 & 36 & 76 & 79 & 170 & 2.2 & 83.7 & 80.9 & 24 \\
\hline
\end{tabular}

*Percentage increase in sites: (number of sites $\left.Y_{r} 2\right) \div\left(\right.$ number of sites in $\left.Y_{r} 1\right) \times 100$ 
one study site address, and first registered after the introduction of the ICMJE policy. Between October 2005 and September 2006, there were 2,597 studies and 74,071 sites registered; between October 2006 and September 2007, there were 2,570 studies and 52,909 sites registered. The explanation for the fewer sites in the second year is that new sites are continuously added for many newly registered trials.

TABLE 1 and FIG. 1 give our ranking of the top 50 countries based on the total number of study sites, as well as ranking based on annual growth, which differ from those presented in REF. 1. Based on our results, Taiwan, Chile, Brazil, Romania, Switzerland and the US had improved rankings of ten positions or more in annual growth of study sites, whereas Estonia,
Portugal, Slovakia, Czech Republic, Lithuania, Peru, Denmark and Japan all slipped down the rankings by 15 positions or more.

Johan P. E. Karlberg is at the Clinical Trials Centre, Li Ka Shing Faculty of Medicine, The University of Hong Kong, 8/F, Clinical Pathology Building, Queen Mary Hospital, 102 Pokfulam Road, Hong Kong SAR,

People's Republic of China.

e-mail: jpekarl@hkucc.hku.hk

1. Thiers, F., Sinskey, A. J. \& Berndt, E. R. Trends in the globalization of clinical trials. Nature Rev. Drug Discov. 7, 13-14 (2008)

2. US National Institute of Health and The US National Library of Medicine (NLM). Clinical Trial Register. NLM web site [online]. < http://www. clinicaltrials.gov > (2008).

3. De Angelis, C. et al. Clinical trial registration: a statement from the International Committee of Medical Journal Editors. N. Engl. J. Med. 351, 1250-1251 (2004).

4. Karlberg, J. P. E. Sponsored clinical trial globalization trends. Clin. Trial Magnifier 1, 13-19 (2008). 